\title{
Analysis of Base Station Assisted Novel Network Design Space for Edge-based WSNs
}

\author{
Muni Venkateswarlu K. and A. Kandasamy \\ Department of Mathematical and Computational Sciences, National Institute of Technology Karnataka, Mangalore, 575 \\ 025, India \\ Email: \{munivenkateshk, kandy\}@nitk.ac.in
}

Chandrasekaran $\mathrm{K}$.

Department of Computer Science and Engineering, National Institute of Technology Karnataka, Mangalore, 575025 , India

Email: kch@nitk.ac.in

\begin{abstract}
Limited and constrained energy resources of wireless sensor network should be used wisely to prolong sensor nodes lifetime. To achieve high energy efficiency and to increase wireless sensor network lifetime, sensor nodes are grouped together to form clusters. Organizing wireless sensor networks into clusters enables the efficient utilization of limited energy resources of the deployed sensor nodes. However, the problems of unbalanced energy consumption exist in intra and inter cluster communication, and it is tightly bound to the role and the location of a sensor nodes and cluster heads in the network. Also, clustering mechanism results in an unequal load distribution in the network. This paper presents an analytical and conceptual model of Energyefficient edge-based network partitioning scheme proposed for wireless sensor networks. Also, it analyzes different network design space proposed for wireless sensor networks and evaluates their performance. From the experimental results it is observed that, with proper network organization mechanism, sensor network resources are utilized effectively to elevate network lifetime.
\end{abstract}

Index Terms-Edge-based Network design spaces, Network Architectures, Wireless Sensor Networks, Energy Consumption, Network Lifetime.

\section{INTRODUCTION}

Wireless Sensor Network (WSN) is a distributed collection of resource constrained tiny nodes capable of operating with minimal user attendance. Rapid development in micro electro mechanical systems (MEMS) technology has provided small sized, low-power and low-cost sensor nodes with the capability of sensing various types of physical and environmental conditions. WSN improves the ability of humans to monitor and control physical locations from faraway places [1]. Since each sensor node works independently without any central control, failure of some nodes does not affect the other network activities. WSN is more reliable and secure when compared with other types of networks. WSN is the backbone for establishing smarter environments. Each sensor node is equipped with one or more low powered sensors, a processor, memory, a power supply, a radio and an actuator [2]. Based on infrastructure, WSNs are of two types: Structured WSNs and Unstructured WSNs. Nodes are deployed in predetermined way in structured WSN, whereas in unstructured WSN they are randomly deployed. Usually, structured WSN has densely deployed sensor nodes which are not easily manageable and unstructured WSN will have limited number of sensor nodes which can be easily managed [3]. Since WSN has the ability to work without any human intervention, the sensor networks are used in hazardous areas like forests, mountain terrains, military battle fields etc. WSNs are applied in many fields like military, agriculture, industry, target tracking, data collection, rescue missions, national security, monitoring disaster prone areas, managing inventories, health care, home security and environmental studies [4], [5], [16], [17].

Organizing wireless sensor networks into clusters enables the efficient utilization of the limited energy resources of deployed sensor nodes. However, the problem of unbalanced energy consumption exists, and it is tightly bound to the role and the location of a sensor node in the network [6]. Although it is important to continue pursuing novel algorithms and protocols to squeeze most out of existing WSN design spaces, it is equally important to explore other new design paradigms for future sensor networks. Research in the recent past focused on exploring resource abundant and unconstrained energy sources exist in WSN. Base station (BS), is one such resource rich component in WSN. Properties like, resource abundance, unlimited processing capability, huge computational ability, bulk-memory, physical location accessibility, and etc., made BS as a powerful asset for WSN. This insight taps into the capabilities of edge base station, which has not been fully exploited in prior efforts. Several edge-based routing protocols [7], [8], [9] for different organization models are proposed in the literature to shift control overhead burden from sensor node to BS. But, none of them are 
successful in achieving balanced energy consumption between intra and inter cluster communication.

This paper presents an analytical and conceptual model of Energy-efficient edge-based network partitioning scheme proposed for wireless sensor networks in [10]. This paper investigates different wireless sensor network design spaces available in the literature. Also, it high lights the pros and cons of each available wireless sensor network organization model.

\section{RELATED WORK}

Many clustering algorithms and cluster based routing protocols are proposed for WSN. In the recent past, many algorithms are proposed towards effective data communication and data processing with optimal resource usage in the WSN. In this section, we review some of the most effective routing algorithms of WSN.

Low Energy Adaptive Clustering Hierarchy (LEACH) [11] is one of the most popular distributed cluster-based routing protocols for WSN. Each node has a certain probability to become cluster head per round, and the task of being a cluster head is rotated between the nodes. LEACH is highly successful in distributing load uniformly across the network. But, its single hop routing does not serve the requirement of real world applications. Also, the randomized $\mathrm{CH}$ selection method of $\mathrm{LEACH}$ fails to maintain uniform load distribution between the cluster heads.

Lindsey and Raghavendra introduced a chain-based clustering routing protocol, PEGASIS [12]. This is considered as an improvement over LEACH routing protocol. The main aim of PEGASIS is to minimize the intra cluster communication overhead of LEACH protocol. The key idea of PEGASIS is to form chains with closed by neighboring nodes using greedy approach. Each chain chooses a leader node to forward data to BS. Like LEACH, PEGASIS is single hop routing protocol. So, this is not a good choice for large scale networks.

To address hot spot problem, Chengfa Li et al., introduced an unequal clustering mechanism, Energy Efficient Unequal Clustering (EEUC) [13] to balance energy consumption between the cluster heads. EEUC form small clusters near base station and the size increases as the distance progress. Thus the cluster heads close to base station preserve energy for inter-cluster communication. The author also proposed an energy aware multi-hop routing protocol for inter-cluster communication in EEUC mechanism.

Sungju Lee et al, have proposed another unequal clustering algorithm, Energy-Efficient Distributed Unequal Clustering (EEDUC) [14] to create distributed clusters in WSN. EEDUC is an extension to EEUC [13] mechanism. Here also, clusters closer to the base station have smaller size than those farther away from the base station. It considers relay traffic for selecting forwarding $\mathrm{CH}$ to forward data towards BS.

Soro and Heinzelman proposed Unequal Clustering Size(UCS) network organization model for WSN [6]. The main aim of UCS is to enhance the network lifetime by distributing the load uniformly among the $\mathrm{CHs}$, whose positions are predetermined. Having BS at center of the network, the $\mathrm{CHs}$ are arranged symmetrically in concentric circles in two levels called, Layers. Respective clusters in their respective layers are of same size and shape with $\mathrm{CHs}$ at center. But, the cluster size and shape differ from layer to layer. The aggregated data from $\mathrm{CHs}$ will be delivered to $\mathrm{BS}$ through $\mathrm{CH}$ to $\mathrm{CH}$ communication.

Hui-hui Mou and Sun introduced multi-hop clustering algorithm, Power-Efficient Zoning Clustering Algorithm for WSN (PEZCA) [15], to extend network lifetime by minimizing energy consumption. It is developed based on two most popular clustering protocols, Low-Energy Adaptive Clustering Hierarchy (LEACH) [11] and Power-Efficient GAthering in Sensor Information Systems (PEGASIS) [12]. PEZCA divides its network into fan-shaped regions placing $\mathrm{BS}$ at center. Each region is considered as a cluster. $\mathrm{CH}$ to $\mathrm{CH}$ data communication delivers data to BS.

Mao and Hou have introduced a novel edge-based routing protocol, called BeamStar [7] for WSNs. The aim of BeamStar is to reduce size and cost of the sensor node. This protocol utilizes infrastructure potential provided by an edge based network to carry out the network operations. It is assumed that, the network is equipped with a directional antenna with power control capabilities. Using this, BS can reach any part of the network to provide control information to sensor nodes by varying its transmission power level and beam width. This shifts the control and network management overhead burden from sensor nodes to BS. The power controlled capability base station scans the complete network with different power transmission levels (Sector number $(\mathrm{SN})$ ) in different angles (Ring Number (RN)) to provide location information for the nodes. With this location information, sensor nodes can en-route sensed data to BS using controlled broadcasting mechanism. The data is forwarded by using simple forwarding rules provided by BS.

Kuong-Ho and Jyh-Ming proposed a routing protocol for edge-based WSNs, called, CHIRON [8]. It is developed based on one of the most popular hierarchical routing protocols, PEGASIS [12]. Also, it uses the same technique of BeamStar [7] to provide location information for the nodes in the network. It outperforms BeamStar with respect to delay time and network lifetime. CHIRON operates in four different phases. First phase is Group construction phase, where the sensing field is divided into smaller groups using BeamStar methodology. The nodes with same Ids form groups. Chain formation phase is the second phase. Here PEGASIS chain formation process is used to construct smaller chains. Leader node election phase is the next phase in CHIRON. Node with maximum residual energy is elected as "Leader node" for the current round. Cluster Head $(\mathrm{CH})$ to Cluster Head communication delivers data to destination node (BS). $\mathrm{CH}$ selection process repeats in round robin fashion. The last phase is data collection and transmission phase. In this phase, whenever an event 
occurs, the sensor nodes sense the data form their surroundings. The sensed data will be collected and aggregated by chain leader. The same is forwarded to BS using multi-hop, leader-by-leader communication. The CHIRON data transmission process is similar to that of PEGASIS [12] protocol.

To overcome the drawbacks of BeamStar [7], Hao-Li Wang and $\mathrm{Yu}$-Yang Chao proposed a routing protocol for edge-based WSNs, Cluster-based BeamStar (CBS) [9]. CBS also uses the same concept of BeamStar to provide location information for sensor nodes with refined sensing process. CBS outperforms BeamStar in efficient usage of power, internode communication and scan time. CBS protocol is explained in three phases. In the first phase, Locating phase, sensing field is scanned using BeamStar mechanism by adjusting the transmission power level. The second phase is, Cluster building up phase. Here it forms clusters with nodes having same Ids. The node with maximum residual energy is elected as Cluster Head $(\mathrm{CH})$, just like in CHIRON [8]. Data transmission is the last phase in CBS. It uses LEACH [11] protocol to carry out data transmission process. In this phase, $\mathrm{CH}$ aggregates the data from the cluster members and forwards the same to BS via inter-cluster head transmission. New round starts with an advertisement if CHs energy falls below the given threshold. The cluster member with greater residual energy announces itself as a new cluster head for the current round.

\section{BASE Station Assisted Novel NetWork Design SPACE FOR EDGE-BASED WSNS}

To overcome the deficits discussed in the above models, a novel network organization scheme, Energyefficient edge-based network partitioning scheme for wireless sensor networks, is proposed in [10]. The main aim of this proposed scheme is to organize the network in such way that, each part of the network gets equivalent number nodes. It is believed that, with proper node distribution, load can be distributed uniformly across the network with consistent cluster size. The network architectural model proposed is explained in this section.

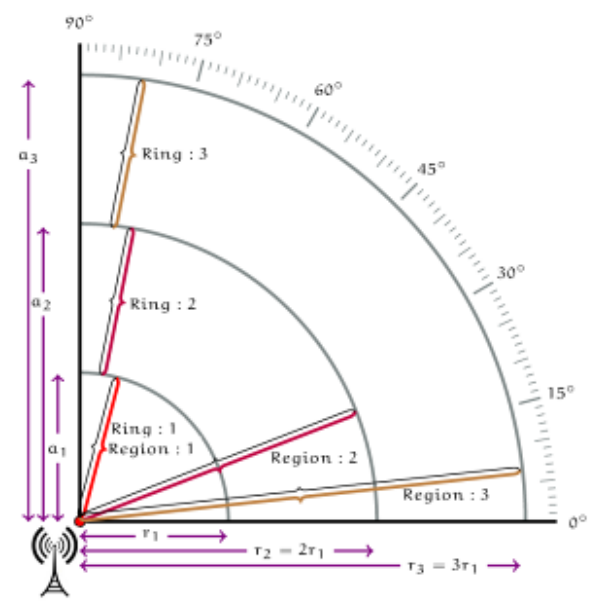

Fig. 1. Region-wise scan of proposed network organization
WSN with $n$ energy constrained sensor nodes deployed randomly in the network field is considered here. BS with Power-controlled directional antenna sits at one corner of the network. Sensor nodes are dispersed within the given radius $r$ from the BS and they have data to send always to destination node with given data rate. This paper highlights the BS role in reducing control overhead on sensor nodes and its importance in network operations.

The proposed method uses the same scan order of CBS [9] to provide location information to sensor nodes in the sensing field. With different transmission power levels $\left(r_{i}\right)$, each ring of the network gets scanned and supplies Ring Number $(R N)$ to sensor nodes. The radius $r_{i}$ for $i^{\text {th }}$ region is chosen as follows.

$$
r_{i}=r \times i, \forall i=1,2,3, \ldots . . n
$$

If any node is scanned more than once, then Received Signal Strength Indication (RSSI) is used to break the tie.

From (3), we get area of $i^{\text {th }}$ region $a_{i}$ as,

$$
r_{i}=\left(\Pi r_{i}^{2}\right) \div 4=i^{2} \times a_{2}, \forall i=1,2,3, \ldots
$$

From (4), we get area of $i^{\text {th }}$ ring $A_{i}$ as,

$$
A_{i}=(2 i-1) \times A_{l}, \forall i=1,2,3, \ldots . . n
$$

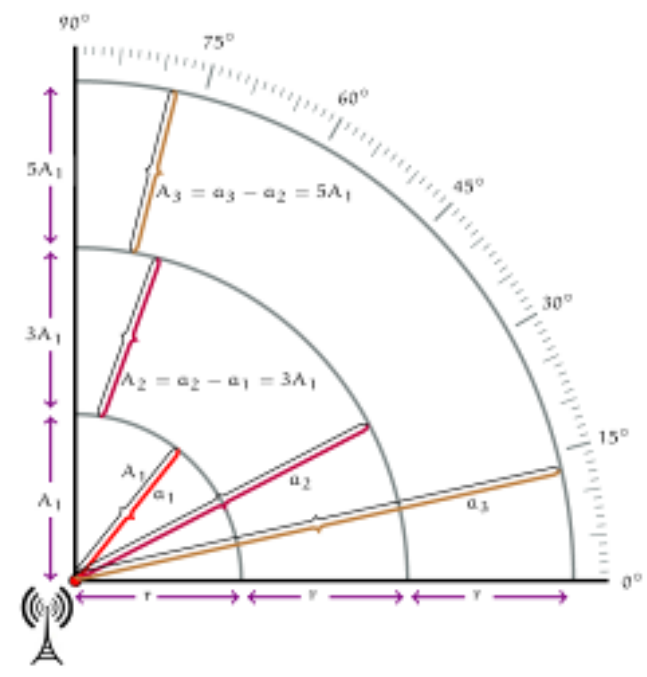

Fig. 2. Areas of each ring in the network

Form (5), every $i^{\text {th }}$ ring can be divide into $(2 i-1)$ equal parts, called, Partitions or Zones. To divide each ring $R_{i}$ into $2 i-1$ parts and to provide sector value, beam-width $(\Theta)$ value is considered for scanning. The $\Theta$ value for $i^{\text {th }}$ ring is chosen as below,

$$
\Theta_{i}=90 \div(2 i-1), \forall i=1,2,3, \ldots
$$

With uniform increment of $\Theta_{i}$ scans $2 i-1$ partitions of ring $R_{i}$. The pair, ring number and partition number, gives identify to each sensor node in the network. $Z_{j}^{i}$ represent $j^{\text {th }}$ partition of $i^{\text {th }}$ ring. 


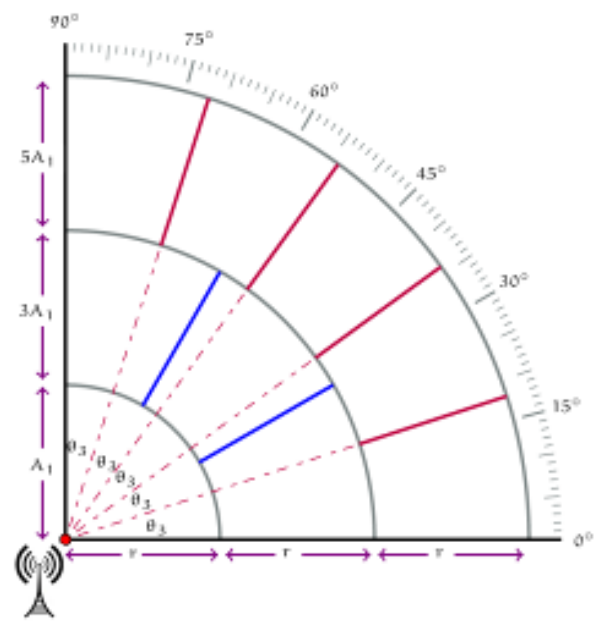

Fig. 3. Sector formation with $\Theta=30^{\circ}$ and $60^{\circ}$

While giving identities to sensor nodes, BS can also provide control information like, forwarding rules, $\mathrm{CH}$ selection strategies, data queries etc.,. Since BS can reach any part of the network it can query any part of the network for sensed information. Without disturbing network activities, BS can update network rules and other control information of a particular network partition. With little overhead, BS can reconfigure all or broken parts of the network.

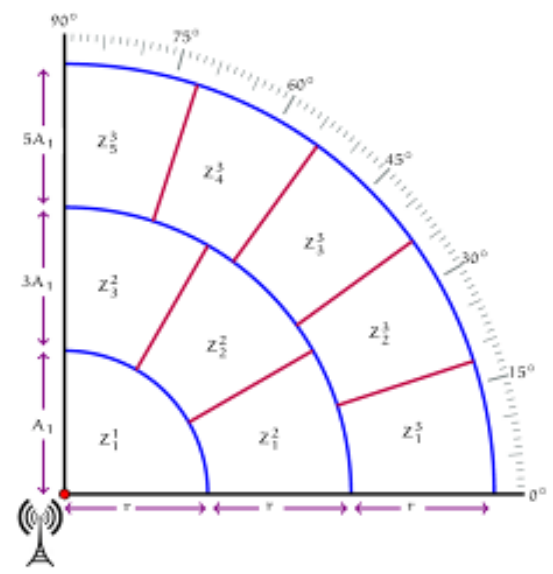

Fig. 4. Proposed novel network architecture

\section{EXPERIMENTAL SECTION}

\section{A. Simulation Environment}

Here we consider, WSN with $n$ sensor nodes deployed randomly within the radius $r$ from the BS. Also, it is assumed that, the BS is equipped with power-controlled directional antenna. As explained earlier, directional antenna scans the network, in order, to provide location information to every sensor node in the network. It is assumed that, the network has data to send always with given data rate.

\section{B. Network Scenarios}

Plain network (Figure 5(a)) with radius $r$, BeamStar network architecture [7] (Figure 5(b)), Cluster-based BeamStar network model [9] (Figure 5(c)) and proposed novel network organization scheme (Figure 5(d)), are four different network scenarios considered for analysis here. BS sits at one corner of the network. Figures 5(a), 5(b), 5(c) and 5(d) illustrate these four network architectures.

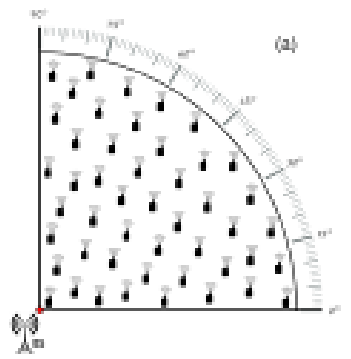

(a). Plain network

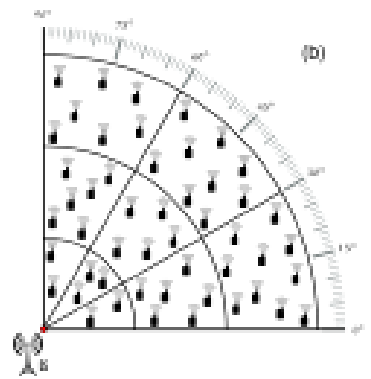

(b). Beam Star network architecture

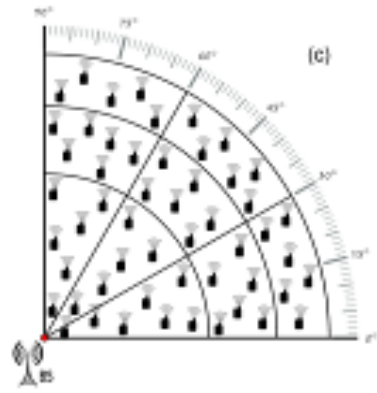

(c). Cluster-based Beam Star network model

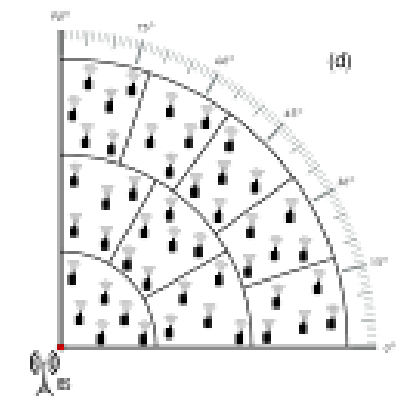

(d). Proposed network design space

Fig. 5: Different network organization mechanisms

\section{Simulation Parameters}

LEACH [11] is one of the most widely accepted routing protocols in the communication world. This protocol is considered here to find best network organization scheme among the above discussed models. $\mathrm{LEACH}$ protocol is experimented on all the four scenarios without losing its originality. Since each 
network scenario has 9 partitions, $9 \mathrm{CHs}$ are elected for each round. Each scenario is simulated for about 5000sec, with 100 and 200 nodes deployed with in 200mts radius from BS. Packet rate takes 1, 5 and 10 values, to observe LEACH behavior on each network scenario given. CASTALIA network simulator [18] is used here to analyze the behavior of LEACH on all the network design spaces considered above.

\section{Simulation Results}

Following figures give total energy consumed and average lifetime of Beamstar, CBS, LEACH and Proposed routing protocol. The results are given by the average value of 10 simulation runs.

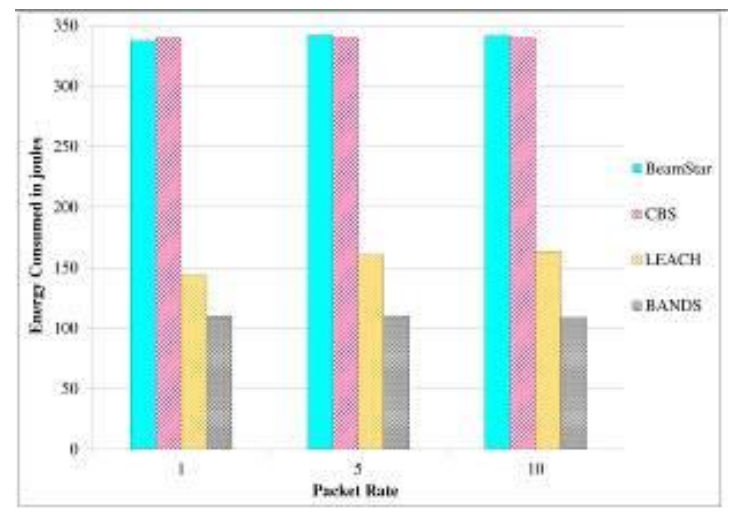

Fig. 6: Total energy consumed for a 100 node network with 50s round length

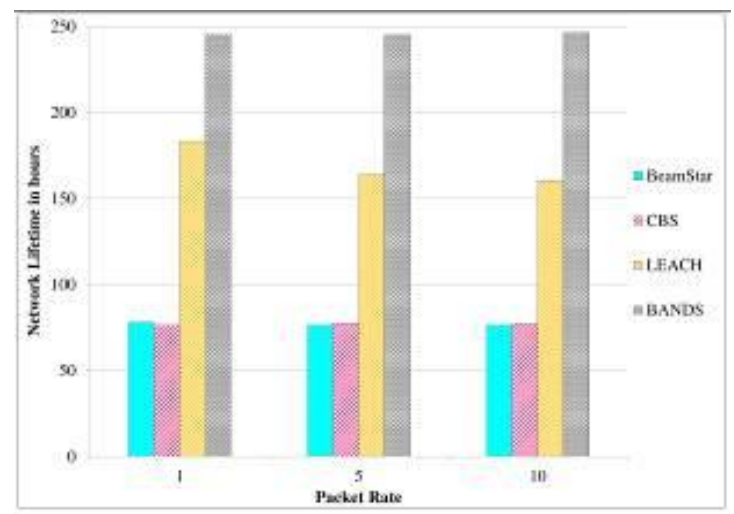

Fig. 7: Average network lifetime for a 100 node network with 50s round length

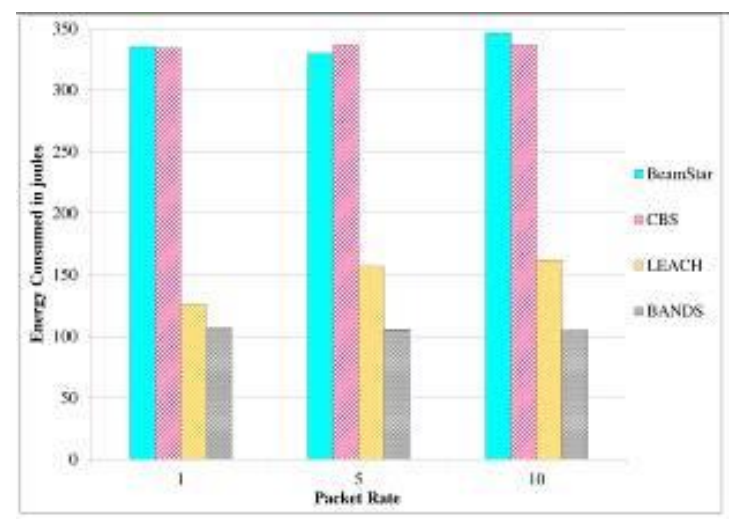

Fig. 8: Total energy consumed for a 100 node network with 100 s round length

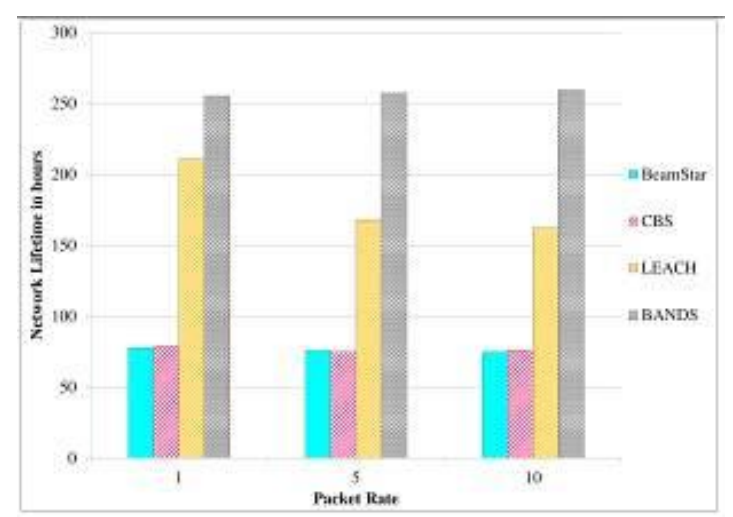

Fig. 9: Average network lifetime for a 100 node network with $100 \mathrm{~s}$ round length

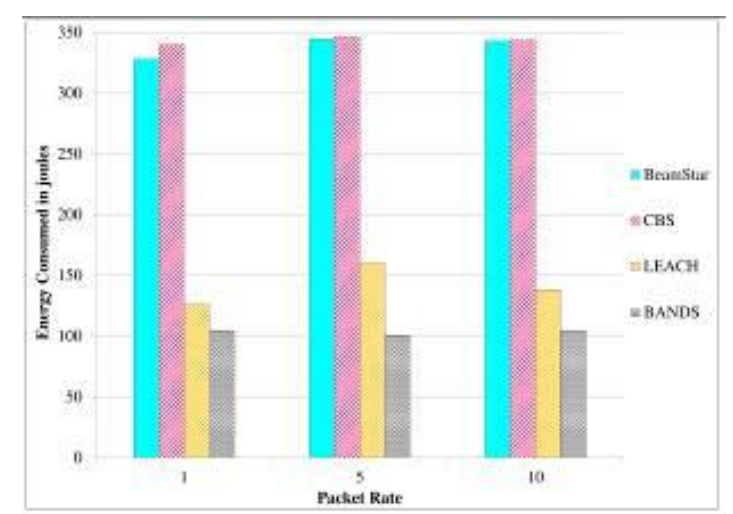

Fig. 10: Total energy consumed for a 100 node network with 250 s round length

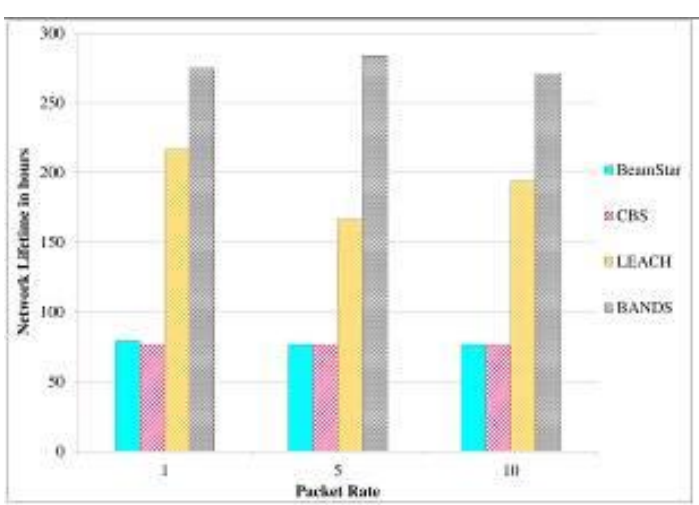

Fig. 11: Average network lifetime for a 100 node network with 250s round length

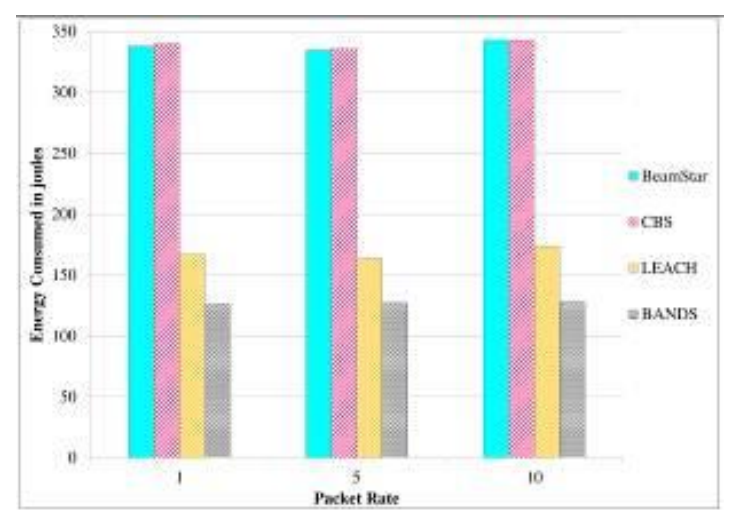

Fig. 12: Total energy consumed for a 200 node network with 50s round length 


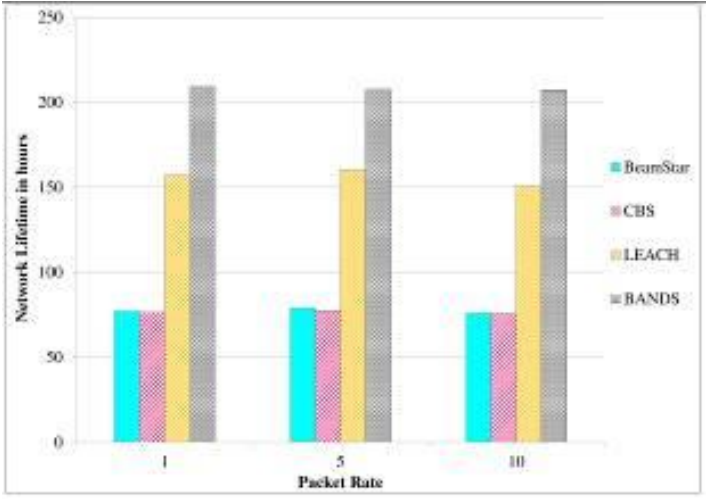

Fig. 13: Average network lifetime for a 200 node network with 50s round length

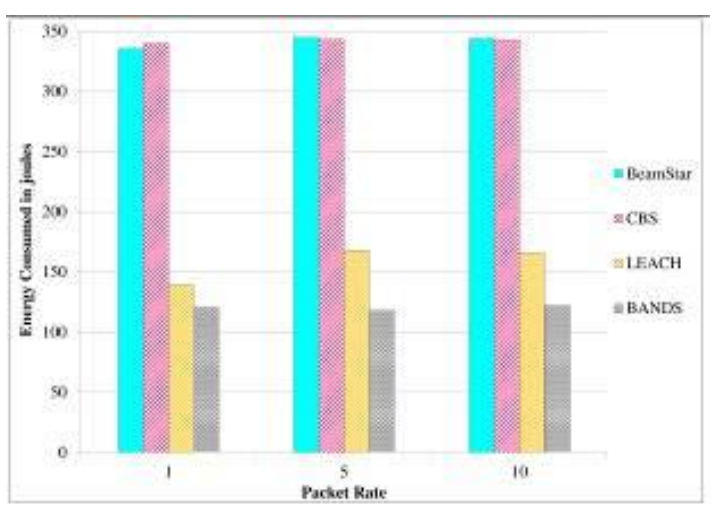

Fig. 14: Total energy consumed for a 200 node network with 100 s round length

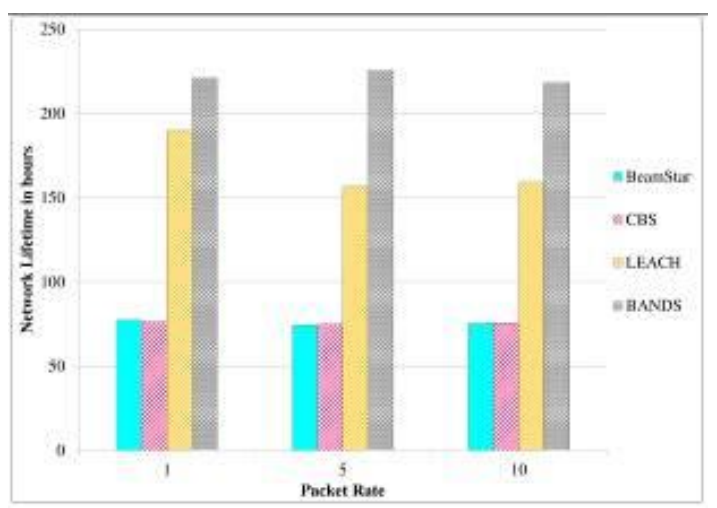

Fig. 15: Average network lifetime for a 200 node network with 100s round length

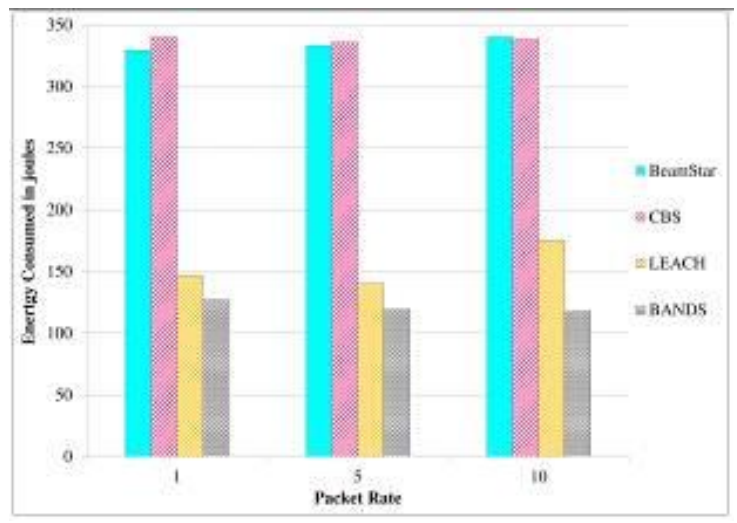

Fig. 16: Total energy consumed for a 200 node network with 250 s round length

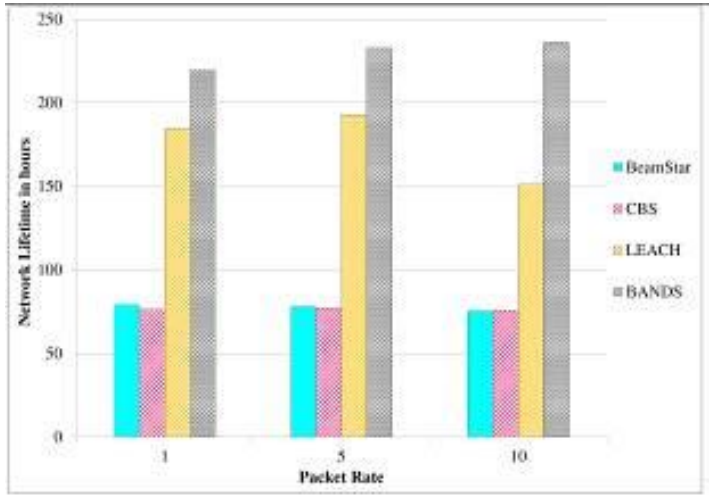

Fig. 17: Average network lifetime for a 200 node network with 250s round length

\section{E. Inference}

From figures 7, 9 and 11, it is clear that the proposed network organization scheme (NNOS-LEACH) improves average network lifetime by atleast $68.1 \%, 68.8 \%$ and $17.5 \%$ against BS-LEACH, CBS-LEACH and LEACH respectively. Also, from figures 6,8 and 10 we note that NNOS-LEACH saves more than $66.8 \%, 67.2 \%$ and $17.4 \%$ of the energy consumption over B-LEACH, CBSLEACH and LEACH respectively in a 100 node network for given data rates.

From figures 13, 15 and 17, it is realized that, NNOSLEACH enhances average network lifetime by minimum of $61.9 \%, 62.7 \%$ and $14.1 \%$ over B-LEACH, CBS-LEACH and LEACH respectively. From figures 12, 14 and 16 it is observed that, NNOS-LEACH conserves $61.6 \%, 62.1 \%$ and $14.8 \%$ of energy against B-LEACH, CBS-LEACH and LEACH respectively in a 200 node network for given packet rates.

Form the above discussion it is observed that, Energy efficient edge-based network partitioning scheme for WSN is highly energy efficient than other network organization models considered. It is also noted that, this model achieves consistency in energy consumption for different data rates. This proves that, this scheme distributes the load uniformly across the network and utilizes minimum energy for network operations. The energy conserved, elevates network availability.

\section{CONCLUSION}

In this paper we evaluate novel network organization scheme, Energy-efficient edge-based network partitioning scheme proposed for WSN in [10]. This work is motivated by exploring the characteristics of resource abundant and un-constrained network resources available in WSN and by analyzing different WSN network organization models that exist in the literature. The proposed model in [10] is successful in conserving sensor nodes energy by distributing load uniformly across the network with consistent cluster size. This is achieved by organizing the network into equal chunks. This helps the network to distribute the load uniformly across the network and saves node energy. Experimental results 
states that, this network organization model consumes less power and elevates network lifetime.

\section{REFERENCES}

[1] D. Bhattacharyya, T.-h. Kim, and S. Pal, "A comparative study of wireless sensor networks and their routing protocols," Sensors, vol. 10, no. 12, pp. 10 506-10 523, 2010. [Online]. Available: http://www.mdpi.com/1424$8220 / 10 / 12 / 10506$

[2] X. Ren and $\mathrm{H}$. Yu, "Multipath disjoint routing algorithm for ad hoc wireless sensor networks," in Eighth IEEE International Symposium on Object-Oriented Real Time Distributed Computing, 2005, pp. 253-256.

[3] J. Yick, B. Mukherjee, and D. Ghosal, "Wireless sensor network survey," Computer Networks, vol. 52, no. 12, pp. 2292-2330, Aug. 2008. [Online]. Available: http://dx.doi.org/10.1016/j.comnet.2008.04.002

[4] K. Akkaya and M. Younis, "A survey on routing protocols for wireless sensor networks," Journal of Ad Hoc Networks, vol. 3, no. 3, pp. 325-349, May 2005.

[5] X. Liu, "A survey on clustering routing protocols in wireless sensor networks," Sensors, vol. 12, no. 8, pp. 11 113-11 153, 2012.

[6] S. Soro and W. B. Heinzelman, "Prolonging the lifetime of wireless sensor networks via unequal clustering," in Proceedings of the 19th IEEE International Parallel and Distributed Processing Symposium (IPDPS'05) Workshop 12, ser. IPDPS '05, vol. 13. Washington, DC, USA: IEEE Computer Society, April 2005, pp. 236-243. [Online]. Available: http://dx.doi.org/10.1109/IPDPS.2005.365

[7] S. Mao and Y. Hou, "BeamStar: An edge-based approach to routing in wireless sensor networks," IEEE Transactions on Mobile Computing, vol. 6(11), pp. 1284 1296, 2007.

[8] C. Kuong Ho, H. Jyh Ming, and H. Chieh Chuan, "CHIRON: An energy efficient chain-based hierarchical routing protocol in wireless sensor networks," in Wireless Telecommunications Symposium (WTS 2009), 2009, pp. $1-5$.

[9] W. H. Li and C. Y. Yang, "A cluster-based data routing for wireless sensor networks," in Proceedings of ICA3PP, LNCS, Springer, vol. 5574, 2009, pp. 129-136.

[10] K. Muni Venkateswarlu, A. Kandasamy, and K. Chandrasekaran, "Energy-efficient edge-based network partitioning scheme for wireless sensor networks," in Proceedings of International Conference on Advances in Computing, Communications and Informatics (ICACCI), 2013, 2013, pp. 1017-1022.

[11] W. R. Heinzelman, A. Chandrakasan, and H. Balakrishnan, "Energyefficient communication protocol for wireless microsensor networks," in Proceedings of the 33rd Hawaii International Conference on System Sciences ser. HICSS '00, vol. 8. Washington, DC, USA: IEEE Computer Society, January 2000, pp. 8020-8029. [Online]. Available: http://dl.acm.org/citation.cfm?id=820264.820485

[12] S. Lindsey and C. Raghavendra, "PEGASIS: powerefficient gathering in sensor information systems," in Proceedings of IEEE Aerospace Conference, no. 3, March 2002, pp. 1125-1130.

[13] C. Li, M. Ye, G. Chen, and J. Wu, "An energy-efficient unequal clustering mechanism for wireless sensor networks," in IEEE International Conference on Mobile Adhoc and Sensor Systems Conference, 2005, 2005, pp. 8 pp. -604 .
[14] S. Lee, J. Lee, H. Sin, S. Yoo, S. Lee, J. Lee, Y. Lee, and S. Kim, "An energy-efficient distributed unequal clustering protocol for wireless sensor networks," World Academy of Science, Engineering and Technology, vol. 48, pp. 443-447, 2008.

[15] F. e. Bai, H. h. Mou, and J. Sun, "Power-efficient zoning clustering algorithm for wireless sensor networks," in Proceedings of International Conference on Information Engineering and Computer Science(ICIECS), 2009, pp. $1-4$

[16] Tarun Dubey and O.P. Sahu, "Omni Directional Antenna Assisted Scheme to Minimize Redundancy in Wireless Sensor Networks", International Journal of Computer Network and Information Security, 2013, 4, pp. 57-62.

[17] P. Raghu Vamsi and Krishna Kant, "An Improved Trusted Greedy Perimeter Stateless Routing for Wireless Sensor Networks", International Journal of Computer Network and Information Security, 2014, 11, pp. 13-19.

[18] A. Boulis, Castalia, A simulator for Wireless Sensor Networks and Body Area Networks, NICTA, Eveleigh, NSW, Australia, October 2013. [Online]. Available: https://github.com/boulis/Castalia.git

\section{Authors' Profiles}

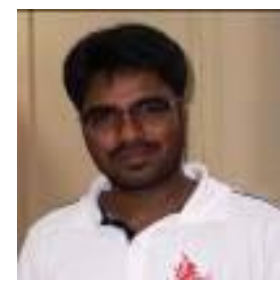

Muni Venkateswarlu $\mathbf{K}$ is a $\mathrm{PhD}$ candidate in the Department of Mathematical and Computational Sciences at National Institute of Technology Karnataka, Mangalore, India. He received master's degree in computer applications from Anna University, Chennai, India and Bachelor's degree from S V University, Tirupati, India. His current research area is wireless sensor networks.

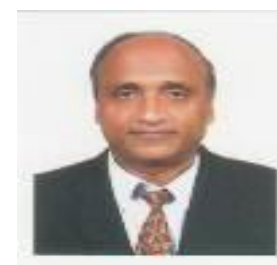

Dr. A. Kandasamy is a professor in the Department of Mathematical and Computational Sciences of National Institute of Technology Karnataka, Surathkal, India. He has done his Doctoral Research at Indian Institute of Technology, Bombay, India and he is a Post-Doctoral Fellow of Chuo University, Tokyo, Japan. His research interests are Computational Fluid Dynamics, Rheology, Tribology, Computational Techniques, Bio-informatics and Wireless Sensor Networks. He has published more than 50 reviewed papers in the reputed international journals and international/national conference proceedings. He has given invited talks in various conferences at national and international levels including the ones held at Russia, U.K., Singapore, Malaysia, Indonesia and Hong Kong. He has guided till now fiver students at Doctoral level research work, more than 25 students at Master's level project work. He is having 23 years of teaching experience and 28 years of research experience. He is Member of Board of Studies of various universities and institutions, Reviewer for various International Journals of Elsevier, Springer, Taylor and Francis and other reputed publications. Dr. Kandasamy is a member of National Board of Accreditation of India. $\mathrm{He}$ is the life member of various Professional Societies at National as well as International levels. At present, he holds the position of Dean of Faculty Welfare at NITK. Mangalore, India. includes the biography here. 


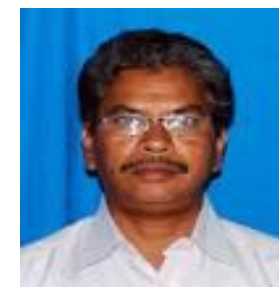

Dr. K. Chandrasekaran is currently Professor in the Department of Computer Science \& Engineering, National Institute of Technology Karnataka, India, having 27 years of experience. He has more than 160 research papers published by various reputed and peer-reviewed International journals, and conferences. He has received best paper awards and best teacher awards. He serves as a member of various reputed professional societies including IEEE (Senior Member), ACM (Senior Member), CSI (Life Member), ISTE (Life Member) and Association of British Scholars (ABS). He is also a member in IEEE Computer Society's Cloud Computing STC (Special Technical Community). He is in the Editorial Team of
IEEE Transactions on Cloud Computing, one of the recent and reputed journals of IEEE publication. He has coordinated many sponsored projects, and, some consultancy projects. He has organized numerous events such as International conferences, International Symposium, workshops and several academic short term programs at NITK. He was a visiting fellow at LMU Leeds, UK in 1995, Visiting Professor at AIT, Bangkok in 2007, and Visitor at UF, USA in 2008 and a Visitor at Univ. of Melbourne, CLOUDS LAB in 2012. He had also worked as Visiting (Professor) at DoMS, IIT Madras during Feb-Dec. 2010. His areas of interest - research include: Computer Communication Networks, Cyber Security and Distributed Computing and Business Computing \& Information Systems Management.

How to cite this paper: Muni Venkateswarlu K., A. Kandasamy, Chandrasekaran K.,"Analysis of Base Station Assisted Novel Network Design Space for Edge-based WSNs", IJCNIS, vol.7, no.7, pp.53-60, 2015.DOI: 10.5815/ijcnis.2015.07.07 